\title{
Adaptive-hierarchical filtering approach for noise removal
}

\author{
Tsung-Nan Lin *, Kai-Jie Chan \\ Department of Electrical Engineering and Graduate Institute of Communication Engineering, National Taiwan University, Taipei, Taiwan
}

Received 7 April 2006; received in revised form 25 July 2007; accepted 8 August 2007

Available online 15 August 2007

\begin{abstract}
In this paper, our contribution is to propose an adaptive-hierarchical filter that can remove the impulse noise while preserving the details in an image. The global image structure, which is estimated from a set of pyramid images, can be used as prior information in order to apply different filters adaptively. The proposed filter outperforms other methods in that it can make use of both local and global information efficiently. Experimental results show that the proposed method produces better performance than many other well-known methods do.
\end{abstract}

(C) 2007 Elsevier B.V. All rights reserved.

Keywords: Adaptive filter; Noise removal

\section{Introduction}

Digital images are often corrupted by impulsive noise due to errors generated in noisy communication channels or sensors [1]. It is essential to remove corrupted pixels before some subsequent processing. Many techniques [25] have been proposed to remove impulse noise from the corrupted images. Median filter [4], which exploits the rank order information of the input data, was initially introduced to effectively remove impulse noise. Despite its effectiveness of noise removal, it tends to modify both noisy pixels and uncorrupted good pixels. Weighted median and center weighted median [3] use a set of weighting parameters in order to preserve more image signal details. Ideally, the filtering should be applied to noisy pixels only. Therefore, various switching techniques are introduced $[2,5-8]$ in order to avoid the damage of good pixels by first classifying image pixels as corrupted and uncorrupted and then passed through the noise-removal filters and identify filters, respectively.

Sun and Neuvo proposed impulse detectors, Switching I and Switching II, with a decision rule based on first order

\footnotetext{
* Corresponding author. Tel.: +8862 33663612; fax: +886223677238.

E-mail address: tsungnan@ntu.edu.tw (T.-N. Lin).
}

statistics by estimating the difference between the current pixel and output of the median filter [5]. Florencio and Schafer introduced a decision measure according to a second order statistic called normalized deviation [8]. Pok et al. proposed the notion of a homogeneity level, which is defined on the basis of the pairwise correlations between neighboring image pixels, to identify the salt and pepper impulse noise [9].

Such kinds of algorithms are proved to be more effective than uniformly applied methods when the noisy pixels are sparsely distributed in the image. However, if images are highly corrupted, a large number of impulse noise may connect into noise blotches. In such cases, it becomes very difficult to detect and eliminate the noise. Additionally, the error will propagate around the nearby regions. Besides, a noise identification scheme is often based on information within a local window so that such an approach is unable to reflect the real global structure of a region. Therefore, the noise cancelation filters are not very effective in preserving the detailed areas.

In this article, our contribution is to propose a novel approach of an adaptive-hierarchical filtering technique to effectively remove the noise while keeping the detailed image information preserved at the same time. The hierarchical filters generate a set of pyramid images. From the 
pyramid structure of an image, the global information of the true image can be effectively differentiated from the noise information. Pixels corrupted by noise in the upper level images will diminish because of the down-sampling process. However, the global information of the image is preserved across all levels of the pyramid.

After estimating the global structure as the prior information, different noise decision filters and error cancelation filters are designed adaptively depending on the global structure of an image. This procedure is applied iteratively to remove noise and preserve the fine detailed information effectively. This iterative reconstruction makes it possible to use partial results as context information to resolve ambiguities in estimating the global structure. The noise pixels processed in the current iteration are used to help better estimate accurate prior information in the subsequent iterations. One main advantage of such an approach is that some impulse pixels located in the middle of large noise blotches can also be properly detected and filtered. Our approach can be illustrated in Fig. 1. Experimental results indicate the proposed algorithm provides better results over many other well-known methods on problems of both uniform random and fixed value (salt-and-pepper) noise.

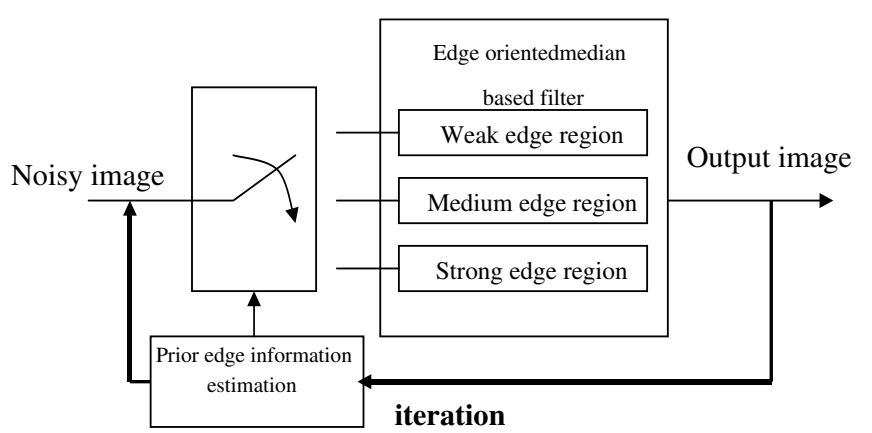

Fig. 1. Adaptive-hierarchical filtering to remove noise iteratively.

\section{Prior information estimation: a hierarchical approach}

It has been well observed that edges contain the most important information of an image. Estimating the true global edge information from a corrupted image is a challenge. In this paper, we propose a hierarchical approach to effectively estimate the true edge.

Using a simple $2 \times 2$ average filter, it is easy to construct a set of pyramid images. It is important to note that the true edge of an image will not disappear in the higher level images while the edges produced by noisy pixels will decrease in the higher level images. Therefore, hierarchical edge detection (HED) scheme shown in Fig. 2 is used to estimate the global true edge information. Sobel edge operators are used [10]. The Sobel operator performs a 2-D spatial gradient measurement on an image and so emphasizes regions of high spatial gradient that correspond to edges. Typically it is used to find the approximate absolute gradient magnitude at each point in an input gray-scale image. Edge value in the upper level image can be set to zero if it is smaller than some predefined values which result from noise. Fig. 3 demonstrates the effectiveness of HED to estimate the structure from a corrupted image. After the estimation, each pixel can be classified as "Weak Edge", "Medium Edge" or "Strong Edge" based on the local statistics. In this paper, we choose to calculate the mean of a $3 \times 3$ window centered about each pixel. Namely,

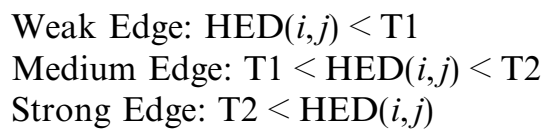

The $\mathrm{T} 1$ and $\mathrm{T} 2$ are the pre-specified thresholds for the above switching operation. T1 and T2 are two thresholds which indicate the fuzziness of the edge information. When the value of HED is greater than T2, it is an indication that the context is a global edge. When the value of HED is in between, it indicates it is median edge. The thresholds are determined based on the statistic data from a lot of images.

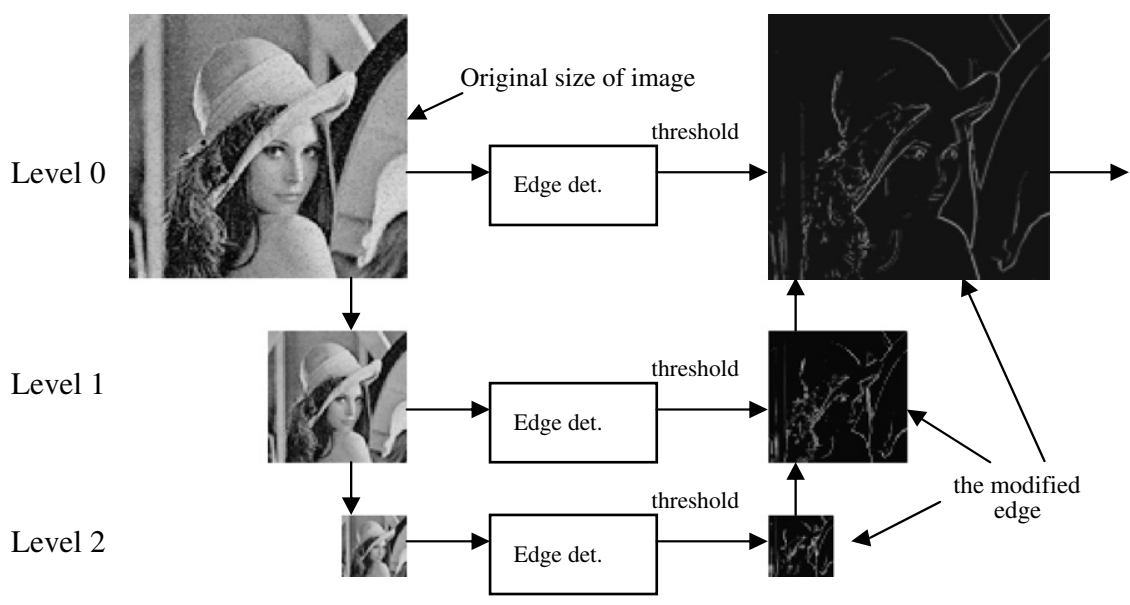

Fig. 2. A hierarchical approach to estimate the image structure. 
a
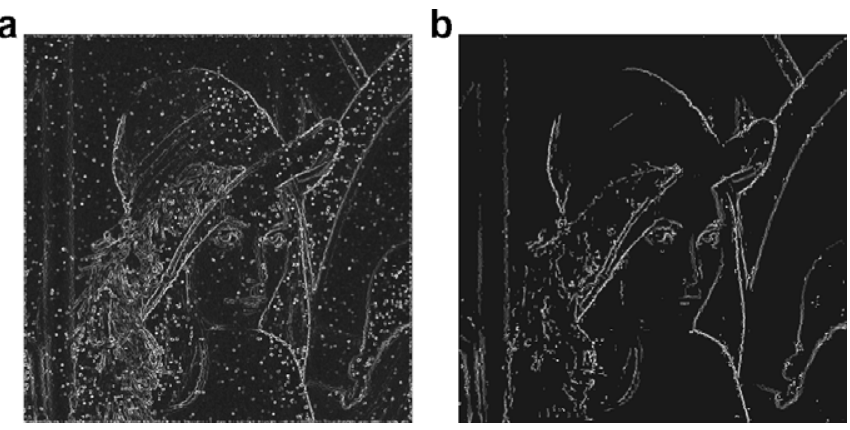

Fig. 3. Comparison of image structure estimation (a) $40 \%$ noise density without HED (b) $40 \%$ noise density with HED. $40 \%$ noise density means $40 \%$ pixels are corrupted by noises.

We choose $\mathrm{T} 1=20, \mathrm{~T} 2=180$ experimentally for a threelayer HED as shown in Fig. 2. The edge value is evaluated within a layer. Since the true edge of an image will not disappear in the higher layer level images while the edges produced by noisy pixels will decrease in the higher level images as shown in Fig. 2. HED is proposed to estimate the prior edge information. Noise decision filters and noise cancelation filters can be designed adaptively according to the global structure of an image after such prior information can be accurately estimated.

\section{Adaptive filters of noise detection and cancelation}

Many switching-based approaches ([8,5]) identify noisy pixels by comparing the output of the median filter or the average value of the filter windows to the value of center pixel. When the distance exceeds a threshold, the center pixel is regarded as a noise pixel. However, using a fixed threshold would lack of adaptivity to noise density variation. Besides, when the noise density is high, a lot of misclassifications will occur and result in more degraded filtering performance.

We apply different noise-detection and noise-cancelation filters according to the image structures adaptively. For pixels belonging to weak and median-edges, noisy pixels are identified based on the distance between the center pixel and the output of a $3 \times 3$ median filter. The threshold is determined adaptively based on the statistics of the local image structure. We compare the distance to the standard deviation of the edge information within the $3 \times 3$ filter. Namely, the noisy pixels in smooth and median-edge regions can be identified as follows:

$\left|x(i, j)-\operatorname{Med}_{i j}\right|>K * \rho_{i j}$

where $\rho_{i j}$ denotes the standard deviation of edge information within the $3 \times 3$ block and $\operatorname{Med}_{i j}$ is the output of the median filter within the block. Eq. (1) identifies noisy pixels by comparing to the local statistics of neighbor pixels. The threshold is not fixed but adaptive to the local image structure. Intuitively, the threshold should be small when the pixels are in the smooth regions. In the median-edge regions, the threshold should be larger to accommodate the local variation in the image texture.

To get a good estimation of the $K$ value, we calculate the local statistics of several uncorrupted images. From experiments, it is noted that $97-99 \%$ pixels $(x(i, j))$ satisfy the condition that $\left|x(i, j)-\operatorname{Med}_{i j}\right|<K^{*} \rho_{i j}$ if the value $K$ is set between 1.5 and 2 for smooth and median-edge regions. Therefore, we choose the value $K$ to be 1.5 and 2 for smooth and median-edge regions, respectively. If the noisy pixel is in the smooth region, it is replaced by the output of the $3 \times 3$ median output. For median-edge region (the information is estimated by HED as shown in Section 2), directional noise-cancelation filters are applied depending on the edge orientation. We utilize the 1-D based median filter $(1 \times 3$ or $3 \times 1$ depending on the edge orientation obtained from the Sobel operator) since it can preserve the edge information better when compared to the 2-D filter.

The third noise detection module distinguishes the considered pixel as non-isolated impulse noise or impulse noise on the edge pixel. Non-isolated impulse noise refers to the considered pixel that belongs to a noise blotch. Noise on the edge can be identified by exploring the property of edge information. Intuitively, it is noted that the generic edge intensity is almost homogeneous along the edge orientation. In other words, if we calculate the variance test of the center pixel $x_{i, j}$ along all possible edge orientations according to Eq. (2), it can easily seen the value will go smaller if the center pixel is a uncorrupted edge pixel. On the other hand, a large value indicates that the center pixel

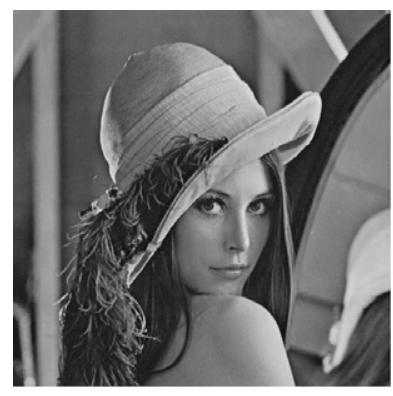

(a) Lena

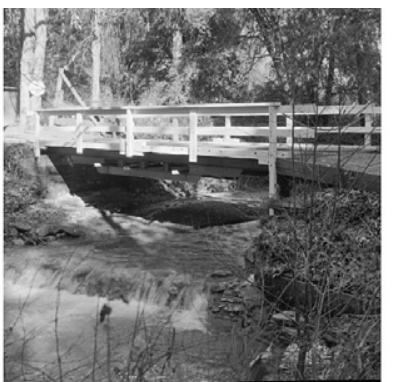

(b) Bridge

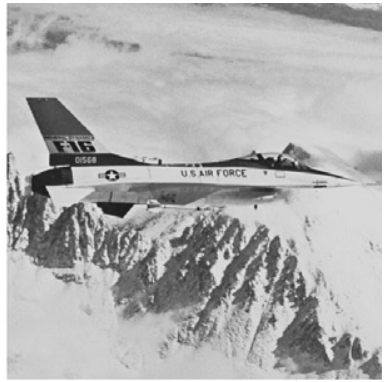

(c) Airplane

Fig. 4. Test images. 
is a corrupted edge pixel. In the following experiments (Section 4), the value is set as $65 \%$.

In essence, the characteristics of a noise blotch (a block of non-isolated noisy pixels) is similar to that of edge pixels since both are high frequency signals. However, they display different characteristics in the pyramid structure. The characteristics of edge pixels behave consistently in the pyramid structure. A noise blotch, on the other hand, becomes an isolated noise, which can be identified based on Eq. (2) similarly, in the upper level images of the pyramid structure. If a pixel is classified as an corrupted edge pixel, the 1 -D based median filter $(1 \times 3$ or $3 \times 1$ depending on the edge orientation obtained from the Sobel operator) is used to remove the noise based on its edge orientation. For a noise blotch, the 2-D median filter of $5 \times 5$ is used.

$d 1=\left|x_{i-1, j-1}-x_{i, j}\right|+\left|x_{i+1, j+1}-x_{i, j}\right|$

$d 2=\left|x_{i-1, j}-x_{i, j}\right|+\left|x_{i+1, j}-x_{i, j}\right|$

$d 3=\left|x_{i-1, j+1}-x_{i, j}\right|+\left|x_{i+1, j-1}-x_{i, j}\right|$

$d 4=\left|x_{i, j-1}-x_{i, j}\right|+\left|x_{i, j+1}-x_{i, j}\right|$

$d_{\min }=\min (d 1, d 2, d 3, d 4)$

\section{Experimental results}

The performance of the proposed algorithm is evaluated based on simulations. Three commonly used gray-scale images as shown in Fig. 4 (Lena, Bridge, and Airplane) are experimented. Fixed-value noise and random-valued noise with uniform distribution are tested. Simulations are carried out for various noise density levels ranging from $10 \%$ to $30 \%$.
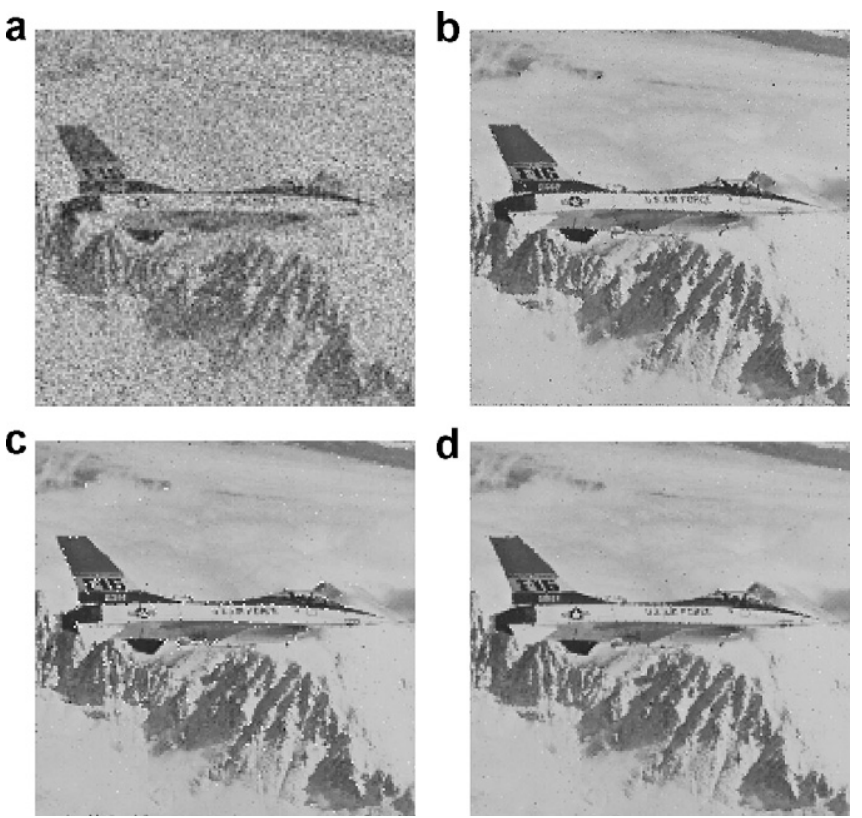

Fig. 5. Iterative restoration performance of airplane image (a) original image corrupted by $20 \%$ random-valued impulse noise. (b) After the first iteration. (c) After the second iteration. (d) Restored image after the third iteration.

Table 1

Restoration results in PSNR for random-valued impulse noise densities ranging from $10 \%$ to $30 \%$

\begin{tabular}{|c|c|c|c|c|c|c|c|c|c|}
\hline \multirow[t]{2}{*}{ Algorithm } & \multicolumn{3}{|l|}{ Lena } & \multicolumn{3}{|c|}{ Bridge } & \multicolumn{3}{|c|}{ Airplane } \\
\hline & $10 \%$ & $20 \%$ & $30 \%$ & $10 \%$ & $20 \%$ & $30 \%$ & $10 \%$ & $20 \%$ & $30 \%$ \\
\hline Median $(3 \times 3)$ & 33.82 & 31.41 & 28.11 & 26.22 & 25.06 & 23.49 & 34.55 & 31.62 & 27.49 \\
\hline$[8]$ & 29.17 & 23.75 & 20.09 & 26.78 & 22.74 & 19.15 & 28.46 & 22.84 & 19.41 \\
\hline Switching I [5] & 35.53 & 32.05 & 28.34 & 26.59 & 25.26 & 23.59 & 35.47 & 32.02 & 27.6 \\
\hline Switching II [5] & 35.89 & 30.44 & 25.66 & 28.53 & 25.73 & 21.35 & 36.09 & 29.74 & 24.73 \\
\hline CWM [3] & 35.17 & 30.32 & 25.66 & 28.26 & 25.64 & 22.91 & 35.74 & 29.73 & 24.76 \\
\hline Proposed & 35.9 & 33.71 & 30.88 & 28.65 & 26.3 & 25.17 & 34.1 & 31.98 & 29.44 \\
\hline
\end{tabular}

Table 2

Restoration results in PSNR for fixed-valued impulse noise densities ranging from $10 \%$ to $30 \%$

\begin{tabular}{|c|c|c|c|c|c|c|c|c|c|}
\hline \multirow[t]{2}{*}{ Algorithm } & \multicolumn{3}{|l|}{ Lena } & \multicolumn{3}{|c|}{ Bridge } & \multicolumn{3}{|c|}{ Airplane } \\
\hline & $10 \%$ & $20 \%$ & $30 \%$ & $10 \%$ & $20 \%$ & $30 \%$ & $10 \%$ & $20 \%$ & $30 \%$ \\
\hline Median $(3 \times 3)$ & 33.65 & 29.57 & 23.89 & 26.22 & 24.57 & 21.59 & 34.54 & 29.73 & 23.79 \\
\hline$[8]$ & 26.09 & 18.59 & 14.27 & 25.30 & 18.16 & 14.00 & 25.44 & 18.12 & 14.00 \\
\hline Switching I [5] & 35.05 & 30.08 & 24.02 & 26.55 & 24.76 & 21.66 & 35.53 & 30.02 & 23.85 \\
\hline Switching II [5] & 34.51 & 25.83 & 20.06 & 28.23 & 23.63 & 19.28 & 34.36 & 25.58 & 19.9 \\
\hline CWM [3] & 33.86 & 25.75 & 20.04 & 27.92 & 23.54 & 19.26 & 34.03 & 25.53 & 19.89 \\
\hline Proposed & 35.5 & 32.3 & 29.8 & 28.0 & 26.6 & 24.6 & 37.8 & 33.2 & 29.5 \\
\hline
\end{tabular}


a
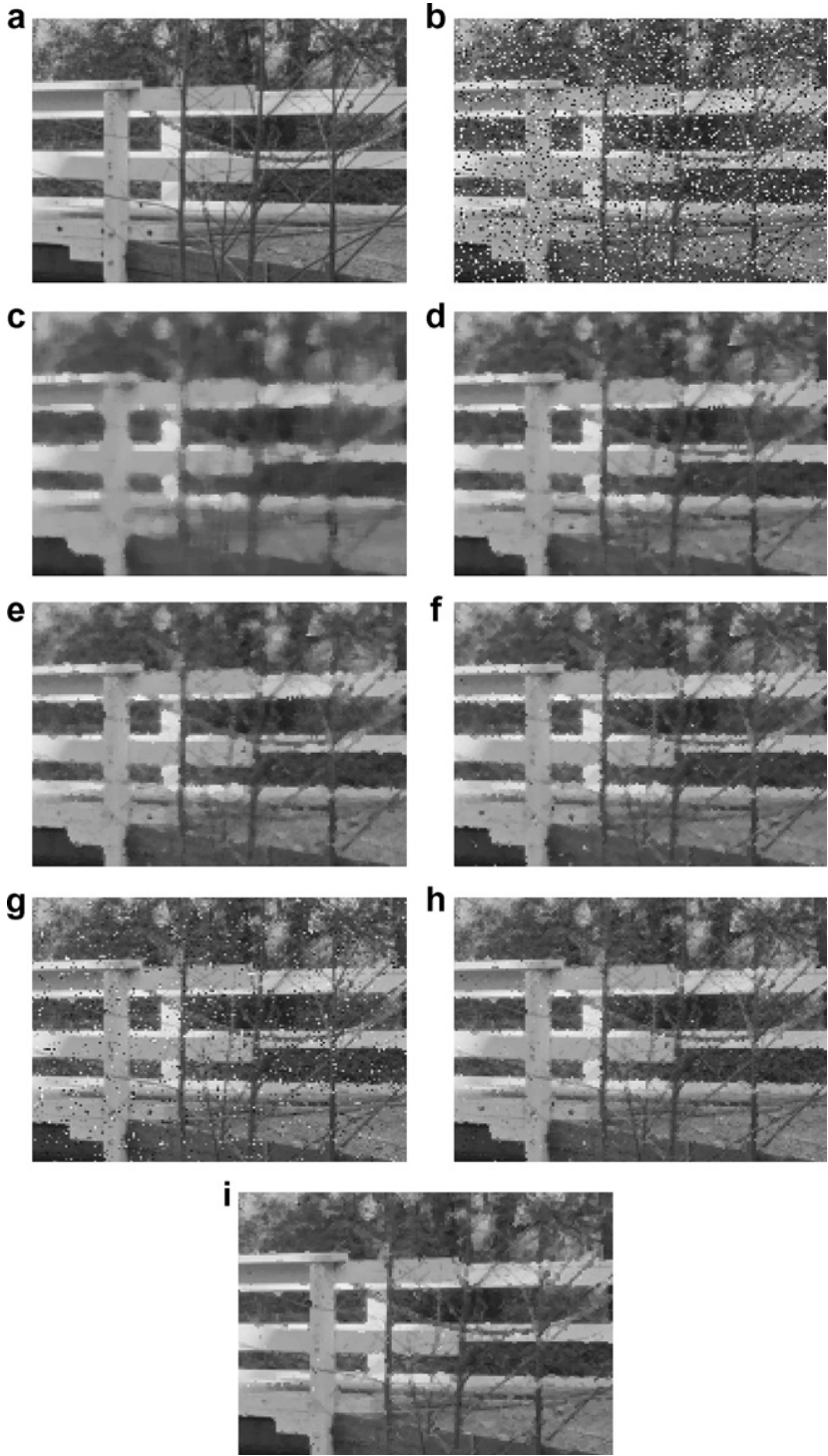

Fig. 6. Restoration results for various methods. (a) Original image. (b) Image corrupted by $20 \%$ random value noise. (c) $5 \times 5$ median filter. (d) $3 \times 3$ median filter. (e) Sun's switching scheme-I. (f) Sun's switching scheme-II. (g) Florencio's filter. (h) MSM filter. (i) The proposed filter.

Peak SNR (PSNR) is used to give a quantitative evaluation. The PSNR performance of the proposed mechanism is compared to that of the $3 \times 3$ median filter, $5 \times 5$ median filter, Florencio and Schafer's switching scheme, Sun and Neuvo's switching scheme-I and scheme-II, $3 \times 3 \mathrm{CWM}$ filter ([3]), MSM filter ([7]), and CSAM ([9]) filter.

Tables 1 and 2 demonstrates the PSNR results for random-valued noise and fixed value noise, respectively. Both simulation results show the proposed filtering outperforms other filtering schemes. We apply three iterations of the proposed to restore the corrupted image. Fig. 5 demonstrates the noise is removed gradually in the interative process since the filtered image in the current iteration provides better estimation of image global structure in the next iteration. The consistency of the performance from Tables 1 and 2 indicates that the proposed filter is fairly robust against wide variations of noise densities.

A subjective visual comparison of the noise reduction and image detail-preserving using "Bridge" image is presented in Fig. 6. We can observe that outputs of Switching I and median filter have the edge blurring effect. On the contrary, even though Florencio and MSM have better edge preserving result, it is observed from Tables 1 and 2 that PSNR will decrease substantially in the high noise density.

\section{Conclusions}

In this paper, we present an adaptive-hierarchical filtering technique to remove the impulse noise effectively while preserving the detailed image information at the same time. The global image structure is estimated as prior information which can be used to apply different filters adaptively. A noisy pixel can be effectively removed by either a standard median filter or the edge directional filter depending on the local statistics of the image structure. The procedure is applied iteratively. The iterative reconstructions make it possible to use partial results as context information to resolve ambiguities in estimating the global structure. The proposed filter outperforms other methods in that it can make use of both local and global information efficiently. Experimental results show that the proposed method produces better performance than many other well-known methods do.

\section{References}

[1] W.K. Pratt, Digital Image Processing, Wiely, New York, 1978.

[2] How-Lung Eng, Kai-Kung Ma, Noise adaptive soft switching median filter, IEEE Trans. Image Process. 10 (2001) 1834-1838.

[3] S.-J. Ko, S.-J. Lee, Center weighted median filters and their applications to image enhancement, IEEE Trans. Circuits Syst. 15 (1991) 984-993.

[4] I. Pitas, A. Venetsanopou, Nonlinear Digital Filters: Principles and Application, Kluwer, Norwell, MA, 1990.

[5] T. Sun, Y. Neuvo, Detail-preserving median based filters in image processing, Pattern Recognit. Lett. 15 (1994) 341-347.

[6] Tao Chen, Kai-Kuang Ma, Li-Hui Chen, Tri-state median filter for image denoising, IEEE Trans. Image Process. 8 (1999) 1834-1838.

[7] Tao Chen, Hong Ren Wu, Space variant median filters for the restoration of impulse noise corrupted images, IEEE Trans. Circuits Syst. II 48 (8) (2001) 784-789.

[8] D.A. Florencio, R.W. Schafer, Decision-based median filter using local signal statistics, Proc. SPIE Int. Symp. Vis, Commun. Image Process. (1994).

[9] Gouchol Pok, Jyh-Charn Liu, A.S. Nair, Select removal of impulse noise based on homogeneity level information, IEEE Trans. Image Process. 12 (2003) 1834-1838.

[10] R. Gonzalez, R. Woods, Digital Image Processing, Prentice Hall, 2007. 